\title{
Integration solutions for reconfigurable multi-standard wireless transceivers
}

\author{
A. Kale, G. Batistell, S. Popuri, V. S. R. Pasupureddi, W. Bösch, J. Sturm
}

\begin{abstract}
This article presents an overview of modern integration concepts for receiver, transmitter and duplexer architectures. Based on innovative design techniques, a system-in-package integrated RF transceiver architecture is proposed as a low-cost low-power alternative to traditional multi-standard transceivers. The proposed transceiver architecture unites advantages of a sub-sampling receiver with tunable low noise amplifier (LNA), a high-efficiency digital power amplifier and an integrated duplexer with on-package passive components in order to fulfill the more and more stringent requirements for wireless systems. In addition to the transceiver architecture, measurement results of building blocks are presented. A LNA circuit implemented in $65 \mathrm{~nm}$ CMOS technology provides a continuously tunable-gain ( $3-23 \mathrm{~dB}$ ) and a tunable frequency band $(4.5 \mathrm{GHz}-5.5 \mathrm{GHz})$ with noise figure of $2 \mathrm{~dB}$ and IIP 3 of $-6.5 \mathrm{dBm}$ at the highest gain. A high Q-factor, on-package transformer, implemented in a core-less 3-metal layer system-in-package substrate, presents a coupling factor of 0.6 and a Q-factor of approximately 30. This provides a low-cost, high-performance alternative solution to integrated matching networks and duplexers.
\end{abstract}

Keywords: RF transceivers; multi-standard; sub-sampling; integrated duplexer; tunable LNA

\section{Integrationskonzepte für konfigurierbare Multi-Standard-Drahtlos-Transceiver.}

Der folgende Artikel gibt einen Überblick über moderne Integrationskonzepte von Hochfrequenz-Transceivern, anhand von spezifischen Beispielen innovativer Empfänger, Sender und Duplexer-Architekturen. Das vorgestellte Konzept nutzt moderne Systemin-Package-Technologien gemeinsam mit Chip-Integration als kostengünstige und energiesparende Alternative zu traditionellen Transceiver-Konzepten. Es vereint einen innovativen Sub-Sampling Receiver mit einem flexibel abstimmbaren Low-Noise Amplifier (LNA), einen effizienten digitalen Leistungsverstärker sowie einen passiven Duplexer, integriert in einer System-in-Package-Technologie. Ergänzend zur Transceiver-Architektur wird die Schaltungsrealisierung des LNA mit kontinuierlich verstellbarer Verstärkung von $3 d B$ bis $23 \mathrm{~dB}$, einer verstellbaren Frequenz von $4.5 \mathrm{GHz}$ bis $5.5 \mathrm{GHz}$, einer Noise Figure von $2 \mathrm{~dB}$ und einer IIP3-Linearität von -6.5 dBm bei höchster Verstärkung präsentiert. Weiters wird ein hochqualitativer Transformator vorgestellt, welcher durch Nutzung einer dreilagigen System-in-Package-Technologie realisiert wurde und einen Qualitätsfaktor von 30 sowie einen Kopplungsfaktor von 0.6 aufweist.

Schlüsselwörter: RF Transceiver; Multi-Standard; Sub-sampling; integrierter Duplexer; flexibler LNA

Received August 31, 2017, accepted November 26, 2017, published online February 21, 2018

(C) The Author(s) 2018. This article is published with open access at Springerlink.com

\section{Introduction}

Modern wireless transceivers require multi-band and multi-standard operation, which leads to complex, bulky and power hungry implementations. An architecture for state-of-the-art multi-standard transceiver is shown in Fig. 1, where several transmitter (TX) and receiver $(\mathrm{RX})$ chains are required in order to fulfill specifications for wireless standards.

With the evolution of wireless systems, even more communication standards are being proposed while maintaining backward compatibility, therefore, cost and complexity of RF transceivers tend to increase. For high-performance communication standards, external off-chip components with optimized performance such as duplexer filter and power amplifier (PA), are necessary to satisfy ever-growing standard requirements. On the contrary, for low-performance battery operated applications such as Internet of things (IOT), lowpower and low-cost implementations are desired.

This article presents a set of innovative design concepts, for receivers, transmitters and system-in-package (SiP) integration, which provides reconfigurability, wide frequency operation, higher power efficiency and low-cost. Based on the presented concepts, an RF transceiver architecture shown in Fig. 2 is proposed. The RX chain is based on a tunable active-balun LNA and a sub-sampling down- conversion mixer with discrete-time signal processing (DTSP). The transmitter is realized as a high-efficiency digital power amplifier (DPA) working as RF-DAC. Finally, a SiP integration provides highquality, low-cost passive components for matching network and duplexer implementation

The whole transceiver architecture is thought to be designed in a single-chip, avoiding cost increase caused by a multi-chip solution.

Kale, Ajinkya, Centre for VLSI and Embedded Systems Technology (CVEST), International Institute of Information Technology Hyderabad, Hyderabad, India; Josef Ressel Center for Integrated CMOS RF Systems and Circuits Design, Carinthia University of Applied Sciences, Europastraße 4, Villach, Austria (E-mail: a.kale@fh-kaernten.at); Batistell, Graciele, Josef Ressel Center for Integrated CMOS RF Systems and Circuits Design, Carinthia University of Applied Sciences, Europastraße 4, Villach, Austria (E-mail: g.batistell@fh-kaernten.at); Institute of Microwave and Photonic Engineering, Graz University of Technology, Graz, Austria; Popuri, Suchendranath, Josef Ressel Center for Integrated CMOS RF Systems and Circuits Design, Carinthia University of Applied Sciences, Europastraße 4, Villach, Austria (E-mail: s.popuri@fh-kaernten.at); Pasupureddi, Vijaya Sankara Rao, Centre for Advanced Studies in Electronics Science and Technology (CASEST), School of Physics, University of Hyderabad, Hyderabad, India; Bösch, Wolfgang, Institute of Microwave and Photonic Engineering, Graz University of Technology, Graz, Austria; Sturm, Johannes, Josef Ressel Center for Integrated CMOS RF Systems and Circuits Design, Carinthia University of Applied Sciences, Europastraße 4 Villach, Austria (E-mail: j.sturm@fh-kaernten.at) 


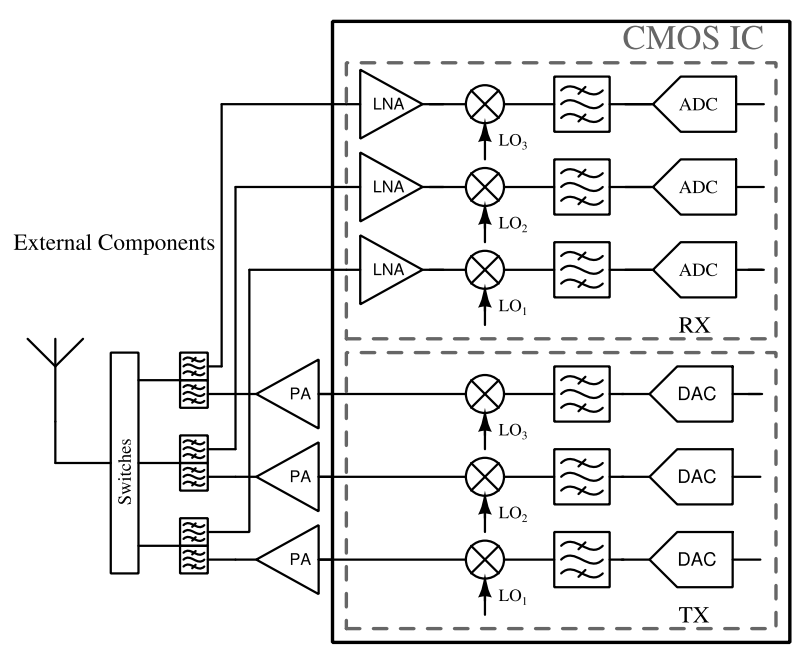

Fig. 1. Traditional multi-standard transceiver architecture with external passive components

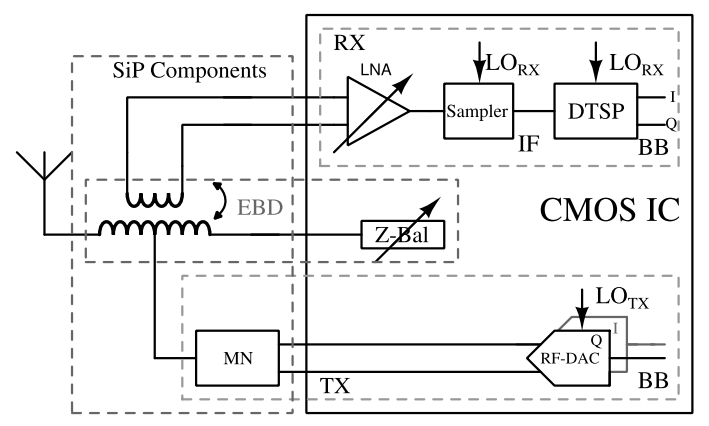

Fig. 2. Proposed transceiver with sub-sampling receiver, SiP integrated electric balance duplexer (EBD) and digital intensive transmitter

A tunable-band LNA relaxes the linearity requirements of wideband LNAs, and the programmable gain fulfills the sensitivity requirements of various wireless standards. Ideal software defined radio (SDR) concept relies on a analog-to-digital converter (ADC) to digitize the RF signal immediately after the antenna and transfer the complete signal processing to the digital domain [1]. For example, for a Nyquist ADC with $2 \mathrm{GHz}$ RF input signal, a sampling frequency of $4 \mathrm{GSps}$ is necessary with high enough resolution. These stringent specifications are currently not practical for battery operated low-power applications. The sampling receivers provide a step towards this goal by employing discrete-time signal processing with additional flexibility $[4,5,11,13]$. Receiver implementations in $[4,13]$ focus on oversampling the RF signal to place the images above the center frequency. The tuned receiver in [11] samples the $2.4 \mathrm{GHz}$ RF signal at a very low sampling frequency of $100 \mathrm{MHz}$ resulting in very high noise figure of $21 \mathrm{~dB}$ due to noise folding. The implementation [5] utilize bandpass sampling theory to optimize the sampling frequency of $1.07 \mathrm{GHz}$ for $2.4 \mathrm{GHz}$ RF signal but lacks the frontend LNA. The proposed sub-sampling receiver architecture utilizes sub-sampling mixer followed DTSP after the front-end LNA. Use of sub-sampling leads to lower local oscillator frequency, less-complex frequency synthesizer and lower power consumption compared to traditional all analog receivers.

An RF-DAC unites in one block the functionality of a digital-toanalog converter (DAC), up-conversion and amplification stages. It

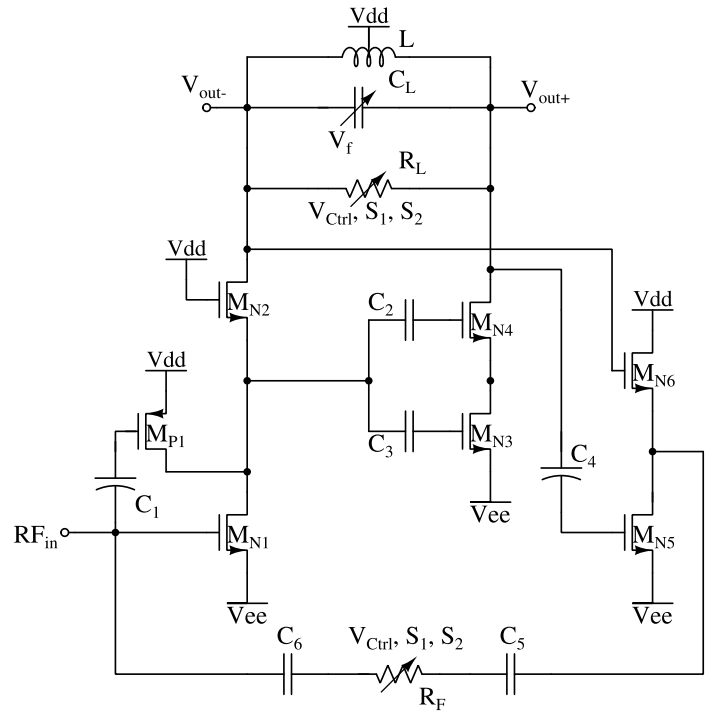

Fig. 3. Tunable-gain and tunable-band active balun LNA circuit diagram with buffered feedback [12]

has the advantage of higher efficiency when compared to traditional analog PAs and can be integrated in standard CMOS technology. An electric balance duplexer (EBD) can be fully integrated in a SiP and prevents the use of external costly duplexer. SiP transformers can be used in the matching network (MN) and Duplexer as an alternative to CMOS transformers. They reduce the production cost and additionally contribute to a more efficient RF-DAC and low loss duplexer structures.

The rest of article is organized as follows, in Sect. 2 a review about sub-sampling receivers will be presented with LNA implementation details. In Sect. 3 a review and analysis of a DPA will be presented. In Sect. 4 implementation and measurement results of on-package transformers for duplexer and MN will be shown. In Sect. 5 recently proposed solutions for integrated duplexers, its advantages and limitations will be discussed. Section 6 presents conclusions and final considerations of the proposed transceiver.

\section{Sub-sampling receiver architecture}

The sub-sampling receiver utilizes a tunable-gain and tunable-band active-balun common-source LNA followed by a sub-sampling mixer that down-converts the RF signal to IF and a DTSP block that downconverts the IF signal to baseband.

The LNA shown in Fig. 3, is based on the buffered resistive feedback topology with noise cancellation techniques as reported in [12]. The LNA closed loop gain is tuned by simultaneously varying the load resistor $\left(R_{L}\right)$ and feedback resistor $\left(R_{F}\right) . R_{L}$ and $R_{F}$ are implemented as active CMOS resistor (ACR) with 4 parallel slices as shown in Fig. 4. The ACR is controlled by an external analog signal $V_{C t r l}$ and two digital signals $S_{1}$ and $S_{2}$. The LC-tank provides a tunable bandpass characteristic from $4.5 \mathrm{GHz}$ to $5.5 \mathrm{GHz}$ using tunable MOS varactors. The inductor is a center-tapped coil with a simulated Q-factor of 13 which guarantees the output voltage balance.

The LNA architecture targeted for $5 \mathrm{GHz}$ WLAN band is fabricated in a $65 \mathrm{~nm}$ CMOS technology with an active area of $0.043 \mathrm{~mm}^{2}$. A continuous monotonous gain tuning from $3 \mathrm{~dB}$ to $23 \mathrm{~dB}$ is achieved for the LNA at $5.5 \mathrm{GHz}$ as shown in Fig. 5a. The noise figure (NF) measurement is shown in Fig. $5 \mathrm{~b}$ and it varies between $2 \mathrm{~dB}$ and $6 \mathrm{~dB}$ for different LNA gains. The third order inter-modulation product $\left(I P_{3}\right)$ is measured to be $-6.5 \mathrm{dBm}$ and $+10 \mathrm{dBm}$ at the highest and lowest gains respectively. 


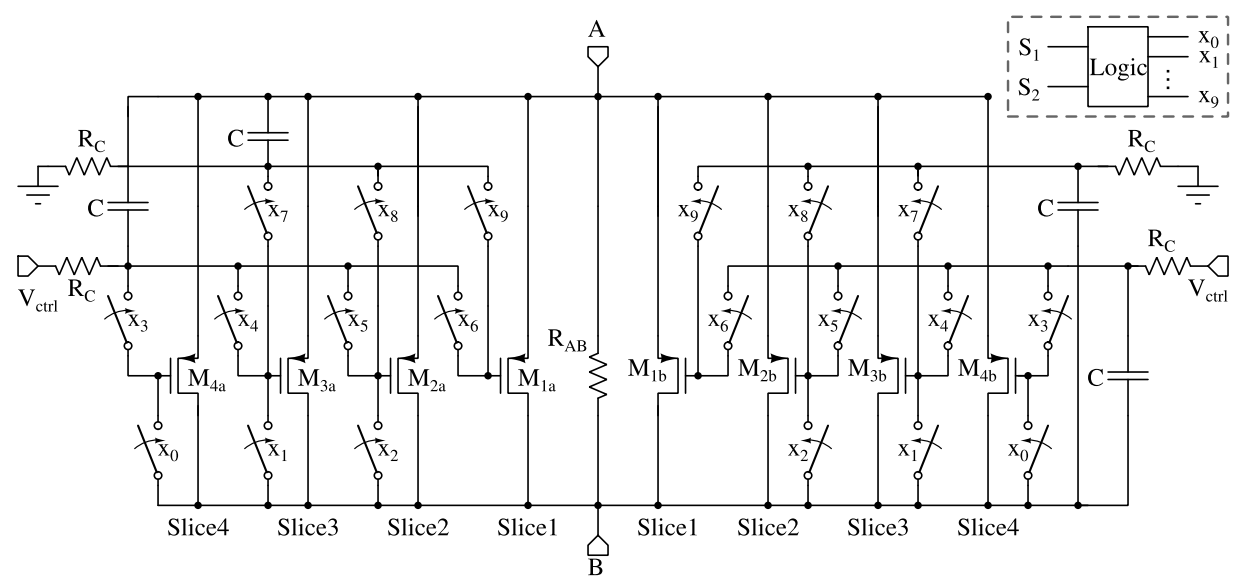

Fig. 4. Active MOS resistor circuit implementation of $R_{F}$ and $R_{L}$ [12]

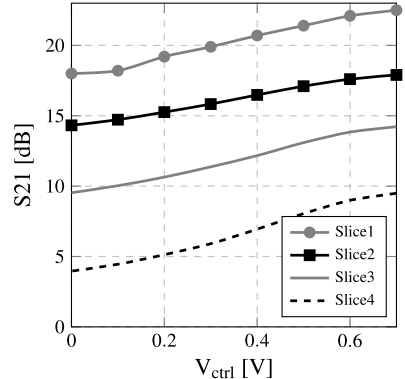

(a) LNA gain sweep

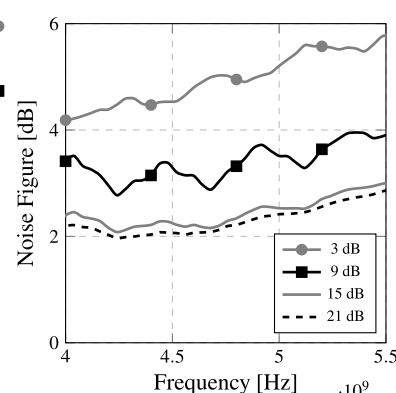

(b) Measured LNA noise figure

Fig. 5. Summary of measured results for tunable-gain and tunable-band LNA [12]

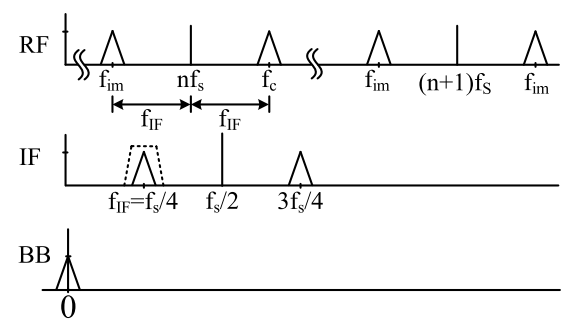

Fig. 6. Frequency spectrum for the sub-sampling receiver with discrete time signal processing [5]

Although the LNA implementation is targeted for $5 \mathrm{GHz}$ WLAN band, the architecture can be modified for lower RF frequency, for example, $2 \mathrm{GHz}$ with similar performance and area penalty of approximately $20 \%$. The proposed receiver architecture utilizes subsampling based down-conversion mixer interfaced to the tunable LNA. The front-end LNA gain reduces the effect of sub-sampling mixer noise figure on the overall receiver noise figure. The LNA-mixer interface must be optimized for the analog tracking bandwidth and $\mathrm{KT} / \mathrm{C}$ noise in the mixer.

The typical spectrum for the sub-sampled RF signal is shown in Fig. 6. Based on the bandpass sampling theory [14], the RF signal with center frequency $f_{c}$ is sampled at sampling frequency of $f_{s}=\frac{4 \times f_{c}}{(2 K-1)}$ and down-converted to an intermediate frequency (IF) $f_{I F}=\frac{f_{s}}{4}$, where $K$ is an integer [5]. The nearest image $\left(f_{i m}\right)$ of this

signal is present at the distance of $\frac{f_{s}}{2}$ as seen in Fig. 6. Selection of sampling frequency is an important parameter for the sampling receivers, based on the effect of noise folding, the lower sampling frequency results in higher noise figure and stringent filter requirement and higher sampling frequency results in easier filter design and lower noise figure, but more complex frequency synthesizer design [14]. If the selected sampling frequency is optimized, the image frequencies can be placed far enough to be filtered before the down-conversion either in the duplexer, in the selectivity of LNA or with a dedicated bandpass filter. Also, the front-end selectivity of the LNA reduces the effect of out-of-band noise on the overall mixer output noise. The second stage down-conversion utilizes discrete time signal processing or discrete time mixing to generate precise in-phase (I) and quadrature phase (Q) signals at the baseband (BB). This approach also has been studied for the wideband receivers in electronic warfare application [6]. The architecture provides a good enough flexibility, low-power design compared to traditional analog receivers.

\section{Digital power amplifier based transmitter}

A key component of a standard wireless transmitter is the PA. Among all the sub-blocks, PA is one of the most challenging blocks due to its requirements of high output power and high-efficiency. Therefore, in most of the cases, the PA is still not integrated into the transceiver chip (Fig. 1). Different semiconductor materials as GaAs and GaN are generally used for its realization, with the advantage of high-performance and drawback of higher production costs. On the other hand, standard CMOS technologies are more affordable than GaAs and GaN, but experience lower supply voltages, limiting the performance of integrated CMOS PAs.

Recently digital power amplifiers (DPAs) have been used as a fully integrated higher efficiency alternative for the conventional analog PAs. An example of DPA is the switched capacitor power amplifier (SCPA) as shown in Fig. 7a [15]. An $n$-bits SCPA consists of a set of $2^{n}$ unit capacitors $C_{u}$ whose bottom plates are switched between $V d d$ and gnd, and top plates are connected in parallel. The number of switching slices corresponds to the number of $O N$-bits. The OFF slices are fixed to gnd or Vdd. The output voltage without a connected load (Vo) is a square wave proportional to the number of switching slices. The switching devices can be implemented as inverters as shown in Fig. 7b. The output impedance of the SCPA can be estimated by the parasitic resistance of the MOS devices $R_{\text {on }}$, the capacitance of $C_{u}$ and the parasitic capacitances $C_{p}$ as shown in 


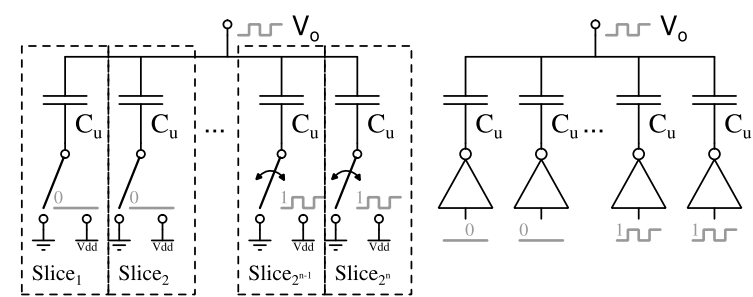

(a)

(b)

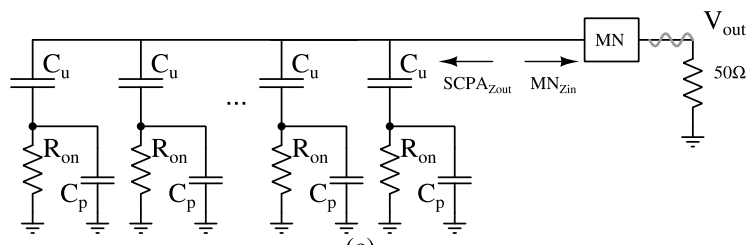

(c)

Fig. 7. Simplified model for switched capacitor power amplifier

Fig. 7c. One advantage of the proposed voltage mode SCPA in comparison to the current mode SCPAs [2] is that the output impedance $S C P A_{\text {zout }}$ is independent of the number of ON and OFF slices and the $\mathrm{MN}$ does not need to be tuned.

The SCPA has an output swing limited by the voltage supply, which is very low in new CMOS technologies. Therefore, a load impedance well below $50 \Omega$ is required to provide a high output power. An impedance matching network is added to the output of the SCPA. For the SCPA implementation, the target maximum efficiency is obtained when the impedance that loads the SCPA $\left(M N_{\text {Zin }}\right)$ has real part larger than $S C P A_{\text {zout }}$ defined by a factor $\alpha$ as show in Eq. (1).

$$
\alpha=\frac{R_{L}}{R_{\text {on }}}=\frac{\text { real } /\left(M N_{\text {Zin }}\right)}{R_{\text {on }}}
$$

The $\alpha$ factor is dependent on the SCPA design and can be empirically defined from a load pull analysis where values between 3 and 6 are generally obtained. The SCPA efficiency analysis is presented in detail in [9] and [15]. The MN provides the correct load impedance for the maximum efficiency, contributes to the impedance matching with the next stage and provides a bandpass characteristic in order to filter the square wave $V_{0}$

Lowering $M N_{\text {Zin }}$ would result in higher SCPA output power. Nevertheless, a low load resistance would require even lower MOS parasitic resistances $R_{o n}$ in order to maintain the ratio $\alpha$. Additionally, challenging large transformation ratios would be necessary on the $\mathrm{MN}$ and a high current would flow through the inverter. Due to these limitations, a standard SCPA with $1 \mathrm{~V}$ supply voltage can achieve peak output power around $18 \mathrm{dBm}$. Most of the cellphone and wireless standards require higher output power, therefore an external PA would still be needed.

Some design techniques can be used to enhance SCPA output power. As SCPA implementation presented in [15], working with stacked devices and higher supply voltages can reach peak output power of $25.2 \mathrm{dBm}$. It uses a $3 \mathrm{~V}$ supply in $1.5 \mathrm{~V}$ technology. High output power can be also obtained by means of power combiner as shown in [10]. This solution can reach peak output power of $28.6 \mathrm{dBm}$ with a standard supply voltage of $1.1 \mathrm{~V}$.

A standard SCPA with low output power can be employed for low-performance, low-range loT application and for highperformance applications such as WLAN and LTE, presented techniques for high output power are necessary. For the proposed fully integrated transceiver, the SCPA implementation provides a lowpower, digital intensive transmitter path.

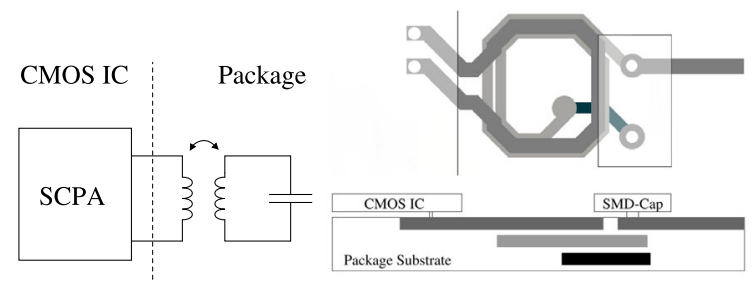

(a) MN schematic

(b) MN top view and cross-section

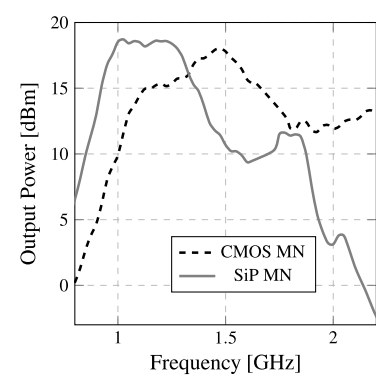

(c) Measured SCPA output power

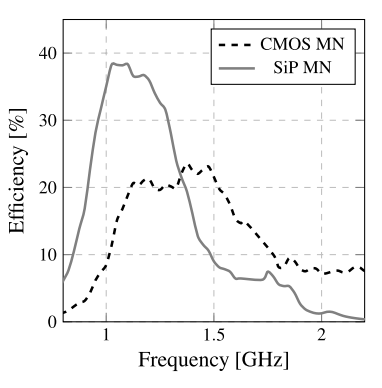

(d) Measured SCPA efficiency
Fig. 8. SiP matching network design and measurement results [3]

\section{Package integration solution}

The impedance MN required for the SCPA is of extreme importance to reach the necessary output power, SCPA efficiency and filtering. For a commonly used differential SCPA, a transformer working as a balun is used as the main component of the MN. The resistive losses added by the parasitics of the transformer strongly influence the overall SCPA efficiency. With the tendency of integrating SCPAs on advanced technology nodes, integrated passives will lose quality and increase cost. Therefore, the research for high-quality low-cost transformers is fundamental for modern RF transceivers.

Recent technologies for system-in-package (SiP) integration are equipped with multiple metals and thin isolation layers, which allow the use of the available package substrate to design low-cost high-quality inductive devices. SiP transformers present a Q-factor of around 30, in comparison Q-factor of 10 to 15 obtained on CMOS technologies. In order to present the feasibility of a SiP MN and its advantages, an implementation example is shown where a $28 \mathrm{~nm}$ CMOS SCPA is evaluated with an on-chip matching network (CMOS-MN) and a package integrated network (SiP-MN).

A SiP-MN was designed on a 3-layer core-less package substrate [3]. As shown in Fig. $8 \mathrm{a}$ and 8b, the MN consists of a balun transformer designed on the substrate metal layers with a capacitor at the secondary, implemented as a surface-mounted device (SMD). The top view and the cross-section view of the MN are presented in Fig. 8b. The measured SCPA output power is shown in Fig. $8 \mathrm{c}$ and the SCPA efficiency in Fig. 8d.

Both implementations reach a peak output power of approximately $18 \mathrm{dBm}$. The SiP-MN presents a peak efficiency of 39\%, which is significantly higher in comparison to the CMOS-MN with $23 \%$. CMOS-MN presents efficiency better than $20 \%$ from $1.1 \mathrm{GHz}$ to $1.5 \mathrm{GHz}$ and SiP-MN presents efficiency better than $30 \%$ from $0.95 \mathrm{GHz}$ to $1.3 \mathrm{GHz}$. It is important to remark that the design of lower frequency matching networks is more challenging, due to lower Q-factor obtained at low frequencies and larger inductance required.

The measured results correspond to a different frequency range than the target application proposed in this article. A redesign can be done for example at $2 \mathrm{GHz}$ center frequency. From the measured results, the transformer Q-factor peaks between $1.5 \mathrm{GHz}$ and $3 \mathrm{GHz}$ 


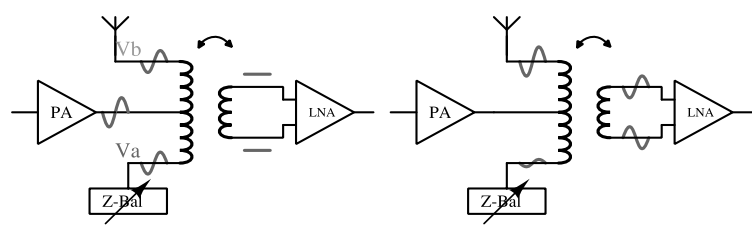

(a) Cancellation of Tx signal

(b) Reception of antenna signal

\section{Fig. 9. Working principle of electric balance duplexer}

which benefits a higher frequency design. Additionally, lower inductor values are required for a higher frequency $\mathrm{MN}$, resulting in area reduction.

\section{Electrically balanced duplexer}

In frequency division duplex (FDD) systems, the RX and TX signals are at neighbor frequency channels with a defined duplex spacing and share the same antenna. In order to ensure that the TX and RX signal do not interfere with each other, high-quality external bandpass filters are used as duplexers, as presented in Fig. 1. Even though many advances have been made in the TX and RX chain, the high-cost external duplexers are still a challenge to overcome.

An electric balance duplexer based on a hybrid transformer and a balanced impedance network was recently proposed as a fully integrated duplexer solution, as presented in Fig. 9 [7].

The PA is connected to the center tap of the transformer's primary. The antenna and the electric balance network $\left(Z_{B a l}\right)$ are connected to the other pins of the primary. $Z_{B a /}$ consists of a tunable impedance network used to mirror the antenna impedance. Ideally, when $Z_{B a}$ impedance matches to the antenna impedance (Fig. 9a), the TX signal flows from the center of the transformer to its extremities creating two identical voltages $\mathrm{Va}$ and $\mathrm{Vb}$. The voltage difference over the transformer's primary is zero, therefore, the signal is not coupled to the secondary, and the receiver is isolated from the TX signal. The RX signal, shown in Fig. 9b, flows from the antenna through the primary and is coupled to the transformers secondary. The tunability of the $Z_{B a l}$ should account for variations of the antenna impedance and environment changes.

$E B D$ is a promising alternative for the high-cost external filter, but it still has several challenges on its implementation. $Z_{B a l}$ needs to be designed for a large tunability range, covering antenna impedance variation of 1.5:1 VSWR, with high-resolution of 32 bits while maintaining the linearity of at least $+70 \mathrm{dBm}$ from TX to the antenna in order to fulfill the full-duplex scenario [8]. Moreover, a symmetrical center-tap transformer causes $3 \mathrm{~dB}$ losses on the TX chain due to the current divider, in addition to the losses due to the low Q-factor of integrated transformers.

The EBD presented in [7] has an isolation larger than the required $50 \mathrm{~dB}$. However, the used hybrid transformer has a low Q-factor of around 10 which results in $11 \mathrm{~dB}$ insertion loss (IL) for the received signal. The insertion loss can be considerably improved with higher Q-factor transformers as shown in [8]. Thicker metal layers and larger area are used to reach a Q-factor around 17 which decreases IL to $3.9 \mathrm{~dB}$.

Large hybrid transformers are feasible when using larger scale less-expensive CMOS technologies. Nevertheless, the large area is a limiting factor for using the EBD in a single-chip transceiver design in advanced technology nodes like $28 \mathrm{~nm}$ and $14 \mathrm{~nm}$.

The transformer quality for SCPA matching network and integrated duplexer is critical as presented in Sects. 3 and 5. These transformers can be implemented on-chip with drawbacks of low Q-factor and a large area in high-cost IC technology. The imple- mented SiP transformers can replace on-chip designs to reduce the production cost, improve the SCPA efficiency and reduce the losses for SiP integrated duplexer.

\section{Conclusions}

In this work, an innovative transceiver architecture is presented based on the principle of sub-sampling down-conversion receiver with tunable-gain tunable-band LNA, high efficiency, fully integrated digital power amplifier (DPA), working as an RF-DAC and System in Package integrated duplexer solutions. Measured results are presented for important building blocks including low noise amplifier and matching network. The DPA provides a digital intensive, highly reconfigurable solution for the transmitter whereas the discrete time signal processing combined with sub-sampling in the receiver provides additional flexibility, low-power and less complex frequency synthesizer design. The presented architecture provides a step forward towards modern wireless front-ends and offers a highly efficient, cost effective solution.

\section{Acknowledgements}

Open access funding provided by Carinthia University of Applied Sciences (CUAS). The presented research work was done in course of the 'Josef Ressel Center for Integrated CMOS RF Systems and Circuits Design - Interact' at the Carinthia University of Applied Sciences. The financial support by the Austrian Federal Ministry of Science, Research and Economy and the National Foundation for Research, Technology and Development is gratefully acknowledged. We also wish to thank Intel Austria GmbH. for their financial contribution and technical support.

Open Access This article is distributed under the terms of the Creative Commons Attribution 4.0 International License (http://creativecommons.org/ licenses/by/4.0/), which permits unrestricted use, distribution, and reproduction in any medium, provided you give appropriate credit to the original author(s) and the source, provide a link to the Creative Commons license, and indicate if changes were made.

\section{References}

1. Abidi, A. A. (2007): The path to the software-defined radio receiver. IEEE J. Solid-State Circuits, 42(5), 954-966.

2. Alavi, M. S., et al. (2013): A $2 \times 13$-bit all-digital I/Q RF-DAC in 65-nm CMOS. In RFIC (pp. 167-170).

3. Batistell, G., et al. (2017): SiP solutions for wireless transceiver impedance matching networks. In European microwave conference (pp. 1068-1072).

4. Chen, R., Hashemi, H. (2014): A 0.5-to-3 GHz software-defined radio receiver using discrete-time RF signal processing. IEEE J. Solid-State Circuits, 49(5), 1097-1111.

5. Jakonis, D., et al. (2005): A 2.4-GHz RF sampling receiver front-end in 0.18- $\mu \mathrm{m}$ CMOS. IEEE J. Solid-State Circuits, 40(6), 1265-1277.

6. Kale, A., et al. (2016): Wideband channelized sub-sampling transceiver for digital RF memory based electronic attack system. Aerosp. Sci. Technol., 51, 34-41.

7. van Liempd, B., et al. (2014): A dual-notch $+27 \mathrm{dBm}$ Tx-power electrical-balance duplexer. In ESSCIRC (pp. 463-466).

8. van Liempd, B., et al. (2016): A > 70-dBm IIP3 electrical-balance duplexer for highly integrated tunable front-ends. IEEE Trans. Microw. Theory Tech., 64(12), 4274-4286.

9. Passamani, A., et al. (2014): A linear model of efficiency for switched-capacitor RF power-amplifiers. In PRIME (pp. 1-4)

10. Passamani, A., et al. (2017): A $1.1 \mathrm{~V} 28.6 \mathrm{dBm}$ fully integrated digital power amplifier for mobile and wireless applications in $28 \mathrm{~nm}$ CMOS technology with 35\% PAE. In ISSCC (pp. 232-233)

11. Pekau, H., et al. (2005): A $2.4 \mathrm{GHz}$ CMOS sub-sampling mixer with integrated filtering IEEE J. Solid-State Circuits, 40(11), 2159-2166.

12. Popuri, S., et al. (2016): A tunable gain and tunable band active balun LNA for IEEE 802.11ac WLAN receivers. In ESSCIRC (pp. 185-188).

13. Ru, Z., et al. (2010): Discrete-time mixing receiver architecture for RF-sampling software-defined radio. IEEE J. Solid-State Circuits, 45(9), 1732-1745.

14. Vaughan, R. G., et al. (1991): The theory of bandpass sampling. IEEE Trans. Signal Process., 39(9), 1973-1984.

15. Yoo, S. M., et al. (2011): A switched-capacitor RF power amplifier. IEEE J. Solid-State Circuits, 46(12), 2977-2987. 


\section{Authors}

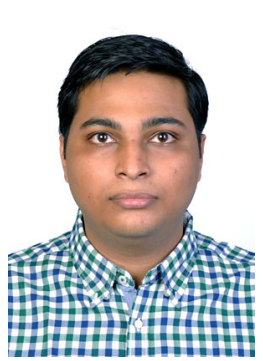

\section{Ajinkya Kale}

received the B.Eng. degree in electronics and communication from Pune University, Pune, India, in 2012. He is currently working as research assistant at the Carinthia University of Applied Sciences (CUAS), Austria and at Josef Ressel Center for Integrated CMOS RF Systems and Circuits Design, Villach, Austria in the field of RF integrated circuits. He is working towards his Ph.D. degree at the Center for VLSI Design and Embedded Systems, International Institute of Information Technology Hyderabad, India under the supervision of Dr. Vijaya Sankara Rao Pasupureddi.

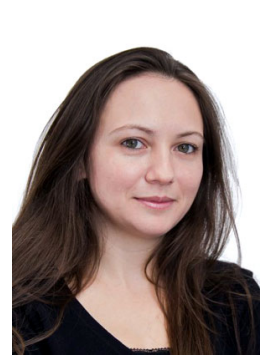

\section{Graciele Batistell}

was born in Concordia, Brasil, in 1985. She received the Bachelor degree in electronic systems from the Federal Institute of Santa Catarina, Florianopolis, Brasil, in 2010, and the Master degree in integrated systems and circuit design program from the Carinthia University of Applied Sciences (CUAS), Villach, Austria, in 2013. In 2011, she joined CUAS as a research assistant. She is currently working as junior researcher and junior lecturer at CUAS in the field of RF integrated circuits and working towards her Ph.D. degree at TU Graz under supervision of Dr. Wolfgang Bösch.

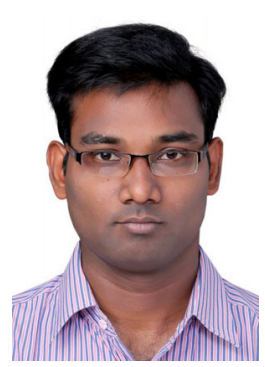

\section{Suchendranath Popuri}

was born in Guntur, India. He received the B. Tech. degree in electronics and communications engineering from VIT University, India in 2007 and the M.Sc. degree in integrated systems and circuits design from the Carinthia University of Applied Sciences, Austria in 2009. He worked as assistant professor in VIT University, India for 3 years from 2010 to 2013. Since July 2013, he has been working as research assistant at Carinthia University of Applied Sciences in the field of RF design. His interests are RF and analog design.

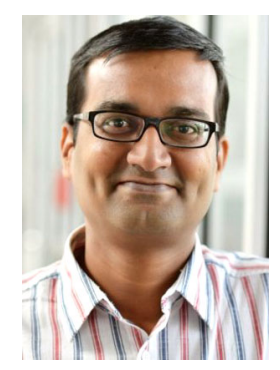

\section{Vijaya Sankara Rao Pasupureddi}

received his Ph.D. degree from the Department of Electronics and Electrical Communication Engineering, Indian Institute of Technology Kharagpur (IIT Kharagpur), India, in 2011. He is an assistant professor at the Centre for Advanced Studies in Electronics Science and Technology (CASEST), School of Physics, University of Hyderabad, India. He was a senior lecturer at The Department of Engineering and IT, Integrated Systems and Circuits Design (ISCD), Carinthia University of Applied Sciences, Austria, and also a senior research associate at Josef Ressel Center for Integrated CMOS RF Systems and Circuits Design, Villach, Austria. He was a post-doctoral scientist at Microelectronic Systems Laboratory (LSM), Swiss Federal Institute of Technology Lausanne (EPFL), Switzerland, from 20122013. He previously worked as an assistant professor at the Indian Institute of Technology Ropar (IIT Ropar) in the Department of Electrical Engineering and IIIT Hyderabad at the Center for VLSI Design and Embedded Systems from 2011-2015. His research interests in- clude analog, RF, and mixed signal integrated circuits and systems for wireless and wireline communications.

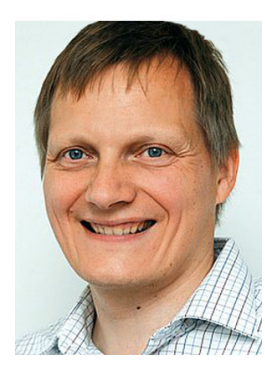

\section{Wolfgang Bösch}

received the Dipl.-Ing. degree in electrical engineering (Hons.) from the Vienna University of Technology, Vienna, Austria, in 1985, the Dr. techn. (Ph.D.) degree (Hons.) from the Graz University of Technology, Graz, Austria, in 1988, and the MBA degree (Hons.) from the Bradford University School of Management, Bradford, U.K., in 2004. In 1987, he joined the European Space Research Centre (ESTEC), Noordwijk, Holland, and worked on different linearization techniques for amplifiers under multicarrier operation (19871989, Research Fellow). In 1989, he was employed by MPR-Teltech, Burnaby, BC, Canada, and was involved in several MMIC technology projects (1989-1991, Senior Engineer). In 1991, he joined the Corporate R\&D group, M/A-COM Inc., Boston, MA, USA (19911993, Principal Engineer). There, he worked on advanced topologies for high-efficiency power amplifiers and on their integration into new technologies. In 1994, he returned to the ESTEC, where he managed several research activities in the field of MMIC technologies, high-efficiency power amplifiers, and new device technologies (HBTs/PHEMTs) (1994-1996, Technical Staff). From 1996 to 1999, he was with DaimlerChrysler Aerospace in Germany, working on T/R modules for airborne radar and space applications (1996-1999, Senior Principal Engineer). From 1999 to 2008, he was with Filtronic plc, Shipley, Saltaire, U.K., where he was the Chief Technology Officer (CTO) of Filtronic Integrated Products and Director of the Global Technology Group. From March 2008 to December 2008, he served as the Director of Business and Technology Integration at RF Micro Devices in the U.K. In 2009, he was the CTO with the Advanced Digital Institute (ADI) in the U.K. In 2010, he joined the Graz University of Technology, to establish the new Institute of Microwave and Photonic Engineering, where he currently serves as the Head of the Institute. He has authored more than 80 papers and holds four patents. Dr. Bösch is a Fellow of the IET. He was a Non-Executive Director of Diamond Microwave Devices (DMD) and ADI. Currently, he is a Non-Executive Director of VIPER-RF (U.K.).

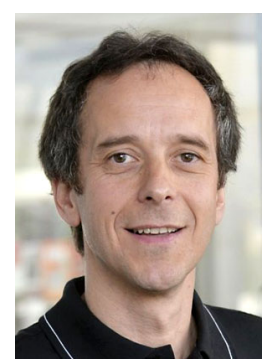

\section{Johannes Sturm}

was born in Villach, Austria. He received the Dipl.-Ing. (M.Sc.) degree in Physics from the Graz University of Technology in 1995 and his Dr. techn. (Ph.D.) degree from Vienna University of Technology in 2006, respectively. In 1996 he joined the Siemens-SemiconductorGroup and later Infineon Technologies in Villach, Austria, working as analog designer, concept engineer and project leader for analog, mixed-signal ASIC projects in the field of optical sensor ICS and high-speed CMOS circuits. Since 2006 he has been employed as Professor for analog circuit design at the Carinthia University of Applied Sciences, Villach, Austria. His research interests are in the field of CMOS RF integrated circuits and integrated sensor design. He has been responsible for several national and European funded research projects and is leading the Josef Ressel Center for Integrated CMOS RF Systems and Circuits Design. He holds 7 patents in the field of microelectronics. 